\title{
THE SELF-GRAVITATING GAS IN THE PRESENCE OF DARK ENERGY: MONTE-CARLO SIMULATIONS AND STABILITY ANALYSIS
}

\author{
H. J. de $\operatorname{Vega}^{(a, b)}$, J. A. Siebert ${ }^{(a)}$ \\ (a) Laboratoire de Physique Théorique et Hautes Energies, \\ Université Paris VI et Paris VII, Laboratoire Associé au CNRS UMR 7589, Tour 24, 5ème étage, \\ 4, Place Jussieu 75252 Paris cedex 05, France. and \\ (b) Observatoire de Paris, LERMA. Laboratoire Associé au CNRS UMR 8112. \\ 61, Avenue de l'Observatoire, 75014 Paris, France.
}

(Dated: October 27, 2018)

\begin{abstract}
The self-gravitating gas in the presence of a positive cosmological constant $\Lambda$ is studied in thermal equilibrium by Monte Carlo simulations and by the mean field approach. We find excellent agreement between both approaches already for $N=1000$ particles on a volume $V$ [The mean field is exact in the infinite $N$ limit]. The domain of stability of the gas is found to increase when the cosmological constant increases. The particle density is shown to be an increasing (decreasing) function of the distance when the dark energy dominates over self-gravity (and vice-versa). We confirm the validity of the thermodynamic limit: $N, V \rightarrow \infty$ with $N / V^{\frac{1}{3}}$ and $\Lambda V^{\frac{2}{3}}$ fixed. In such dilute limit extensive thermodynamic quantities like energy, free energy, entropy turn to be proportional to $N$. We find that the gas is stable till the isothermal compressibility diverges. Beyond this point the gas becomes a extremely dense object whose properties are studied by Monte Carlo.
\end{abstract}

\section{Contents}

I. Introduction 1

II. The self-gravitating gas in the presence of the dark energv

A. Non-relativistic selfgravitating particles in the presence of the cosmological constant 2

B. Statistical mechanics of the self-gravitating gas with $\Lambda \neq 0$

C. The isothermal sphere with $\Lambda \neq 0 \quad 4$

III. Stability of the self-gravitating gas 5

A. Stabilitv for $\Lambda=0$.

$\begin{array}{ll}\text { B. Jeans instabilitv for } \Lambda \neq 0 & 7\end{array}$

\begin{tabular}{lr}
\hline IV. Physical Picture & 7
\end{tabular}

$\begin{array}{ll}\text { A. Behaviour of the gas phase with X } & 7\end{array}$

B. The particle density $\rho(R)]$

\begin{tabular}{|lr}
\hline V. Monte Carlo calculations & 9
\end{tabular}

A. The Metropolis algorithm 9

$\begin{array}{ll}\text { B. Influence of the geometrv } & 10\end{array}$

$\begin{array}{ll}\text { C. Phase diagrams } & 10\end{array}$

$\begin{array}{lr}\text { D. The collapse point } & 10\end{array}$

$\begin{array}{ll}\text { E. Average distribution of particles } & 11\end{array}$

$\begin{array}{ll}\text { F. The condensed phase } & 12\end{array}$

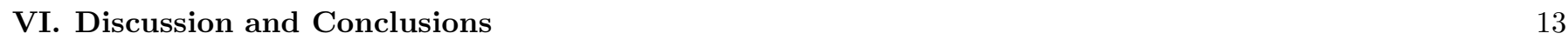

References 14

\section{INTRODUCTION}

The self-gravitating gas in thermal equilibrium has been thoroughly studied since many years 2, 3, 5, 6, 7, 8, 9, 10, 14, 15]. As a consequence of the long range attractive Newton force, the selfgravitating gas admits a consistent 
thermodynamic limit $N, V \rightarrow \infty$ with $\frac{N}{V^{\frac{1}{3}}}$ fixed. In this limit, extensive thermodynamic quantities like energy, free energy, entropy are proportional to $N[2,3]$.

In ref. 4] we investigated how a cosmological constant affects the properties of the non-relativistic self-gravitating gas in thermal equilibrium by mean field methods. The mean field approximation becomes exact in the limit when the number of particles becomes infinity.

In the present paper we study the stability properties of the self-gravitating gas in the presence of a cosmological constant by mean field and Monte-Carlo methods. The use of Monte-Carlo simulations is particularly useful since they are like real experiments and allow to unambiguously determine the stability or instability of the system.

We compute both by Monte Carlo and mean field methods several physical quantities: the equation of state, the particle density and the average particle distance for different values of the dark energy. An excellent agreement between both approaches is found except very close to the collapse transition [see figs. 4-6]. The difference between both approaches turns to be, as expected, of the order $\frac{1}{N}$ where the number of particles $N$ we choose in the Monte Carlo simulations was $N=1000-2000$. The slightly larger split between Monte Carlo and mean field near the collapse is due to the fact that there the corrections to the mean field become singular [2, 3].

We find that the onset of instability in the canonical ensemble coincides with the point where the isothermal compressibility diverges. At this point we find that the dimensionless parameter $\zeta=\frac{G^{3} m^{6} N^{2} P}{T^{4}}$ is maximal. $(\zeta$ was introduced in ref. [10] for $\Lambda=0$ ). We find that the domain of stability of the gas increases for increasing cosmological constant. The dark energy has an anti-gravity effect that disfavours the collapse pushing apart the particles.

In absence of cosmological constant the particle density $\rho(r)$ is a decreasing function of the distance in the selfgravitating gas (see for example 2]). In the presence of a positive $\Lambda$ we find that $\rho(r)$ decreases with $r$ when the self-gravity dominates over the dark energy. This happens for $X \equiv \frac{2 \Lambda V}{m N}<1$. For $X>1$ the cosmological constant dominates over the self-gravity and the the particle density turns to increase with the distance. This is a consequence of the repulsive character of the dark energy.

The Monte-Carlo study of the self-gravitating gas shows the validity of the dilute thermodynamic limit introduced in refs. 2, 4]. Namely, for $N, V \rightarrow \infty$ with $\frac{N}{V^{\frac{1}{3}}}$ and $\Lambda V^{\frac{2}{3}}$ fixed, a consistent thermodynamic limit is reached where extensive thermodynamic quantities like energy, free energy, entropy are shown to be proportional to $N$. Furthermore, the Monte-Carlo simulations allow us to study the condensed phase which turns to be an extremely dense body. The phase transition to collapse is found to be of zeroth order.

The outline of the paper is as follows. Section II presents non-relativistic selfgravitating particles in the presence of the cosmological constant, their statistical mechanical treatment and the mean field approach. In section III we analyze the stability of the selfgravitating gas for zero and nonzero cosmological constant $\Lambda$ while in section IV we give the physical picture of the gas and its particle density as a function of $\Lambda$. Section $\mathrm{V}$ contains the results of our Monte-Carlo simulations on the phase diagram, the collapse transition, the particle density and the properties of the collapsed phase.

\section{THE SELF-GRAVITATING GAS IN THE PRESENCE OF THE DARK ENERGY}

\section{A. Non-relativistic selfgravitating particles in the presence of the cosmological constant}

We recall some results about the self-gravitating gas in the presence of the cosmological constant 44]. In the Newtonian limit the Einstein equations of general relativity become 1]

$$
\nabla^{2} V=4 \pi G \rho-8 \pi G \Lambda
$$

where $V$ stands for the gravitational potential, $\rho$ for the density of massive particles and $\Lambda$ for the cosmological constant. Eq. 2.1) determines the weak and static gravitational field produced by non relativistic matter in the presence of the cosmological constant. For zero cosmological constant we recover the usual Poisson equation, as it must be.

The Hamiltonian for such set of self-gravitating particles in the presence of the cosmological constant can be written in the center of mass frame as $[4$

$$
H=\sum_{i} \frac{\vec{p}_{i}^{2}}{2 m_{i}^{2}}-G \sum_{i<j} \frac{m_{i} m_{j}}{\left|\vec{q}_{i}-\vec{q}_{j}\right|}-\frac{4 \pi G \Lambda}{3} \sum_{i} m_{i} \vec{q}_{i}^{2}
$$

where $m_{i}$ stands for the mass of the particle at the point $\vec{q}_{i}$ with momentum $\vec{p}_{i}$ and

$$
\sum_{i} m_{i} \vec{q}_{i}=0
$$


since we choose center of mass coordinates.

The cosmological constant contribution to the potential energy grows negative for increasing values of the particle distances $\vec{q}_{i}$ to the center of mass. Therefore, the gravitational effect of the cosmological constant on particles amount to push them outwards. It can be then said that the cosmological constant has an anti-gravitational effect.

\section{B. Statistical mechanics of the self-gravitating gas with $\Lambda \neq 0$}

We present here the statistical mechanics of the self-gravitating gas in the presence of the cosmological constant in the canonical ensemble. For simplicity we shall consider $N$ particles with identical mass $m$.

The Hamiltonian is given by eq.(2.2) and therefore the classical partition function of the gas is then,

$$
Z(T, N, V)=\frac{1}{N !} \int \ldots \int \prod_{l=1}^{N} \frac{\mathrm{d}^{3} \vec{p}_{l} \mathrm{~d}^{3} \vec{q}_{l}}{(2 \pi)^{3}} e^{-\frac{H}{T}}
$$

It is convenient to introduce the dimensionless coordinates $\vec{r}_{l}=\frac{\vec{q}_{l}}{L}$. The momenta integrals are computed straightforwardly. Hence, the partition function factorizes as the partition function of a perfect gas times a coordinate integral.

$$
Z=\frac{V^{N}}{N !}\left(\frac{m T}{2 \pi}\right)^{3 N / 2} \int \ldots \int \prod_{l=1}^{N} \mathrm{~d}^{3} \vec{r}_{l} e^{\eta u_{P}+\frac{2 \pi}{3} \xi u_{N}}
$$

where

$$
u_{P} \equiv \frac{1}{N} \sum_{1 \leq i<j \leq N} \frac{1}{\left|\vec{r}_{i}-\vec{r}_{j}\right|} \quad, \quad u_{N} \equiv \sum_{i=1}^{N} r_{i}^{2}
$$

The dimensionless parameters which characterize the strength of self-gravity and dark energy are respectively,

$$
\eta \equiv \frac{G m^{2} N}{T L} \quad \text { and } \quad \xi \equiv 2 \Lambda G m \frac{L^{2}}{T} .
$$

$\eta$ can be interpreted as the ratio of the self-gravitating energy per particle to the kinetic energy per particle.

We define the ratio of dark energy and self-gravity

$$
X \equiv \frac{\xi}{\eta}=\frac{2 \Lambda V}{m N}
$$

It is the ratio between the dark energy contained inside the volume $V$ and the mass of ordinary matter.

In the large $N$ limit the $3 N$-uple integral in eq. (2.3) can be approximated by a functional integral over the density. In the saddle point approximation we find that the density

$$
\rho(\mathbf{x})=e^{\Phi(\mathbf{x})}
$$

obeys to the differential equation [4]

$$
\nabla^{2} \Phi(\mathbf{x})+4 \pi\left(\eta e^{\Phi(\mathbf{x})}-\xi\right)=0
$$

The density has to be normalized as

$$
\int \mathrm{d}^{3} \mathbf{x} \rho(\mathbf{x})=1
$$

In hydrostatic equilibrium we obtain the same equation [4]. Therefore, hydrostatics and mean field are equivalent in the $N \rightarrow \infty$ limit.

In the limiting case $X=0$ eq.(2.8) becomes the well known Lane-Emden equation in the absence of cosmological constant (see refs. [5, 6, 7, 8, 9, 10, 11, 12, 13]). 


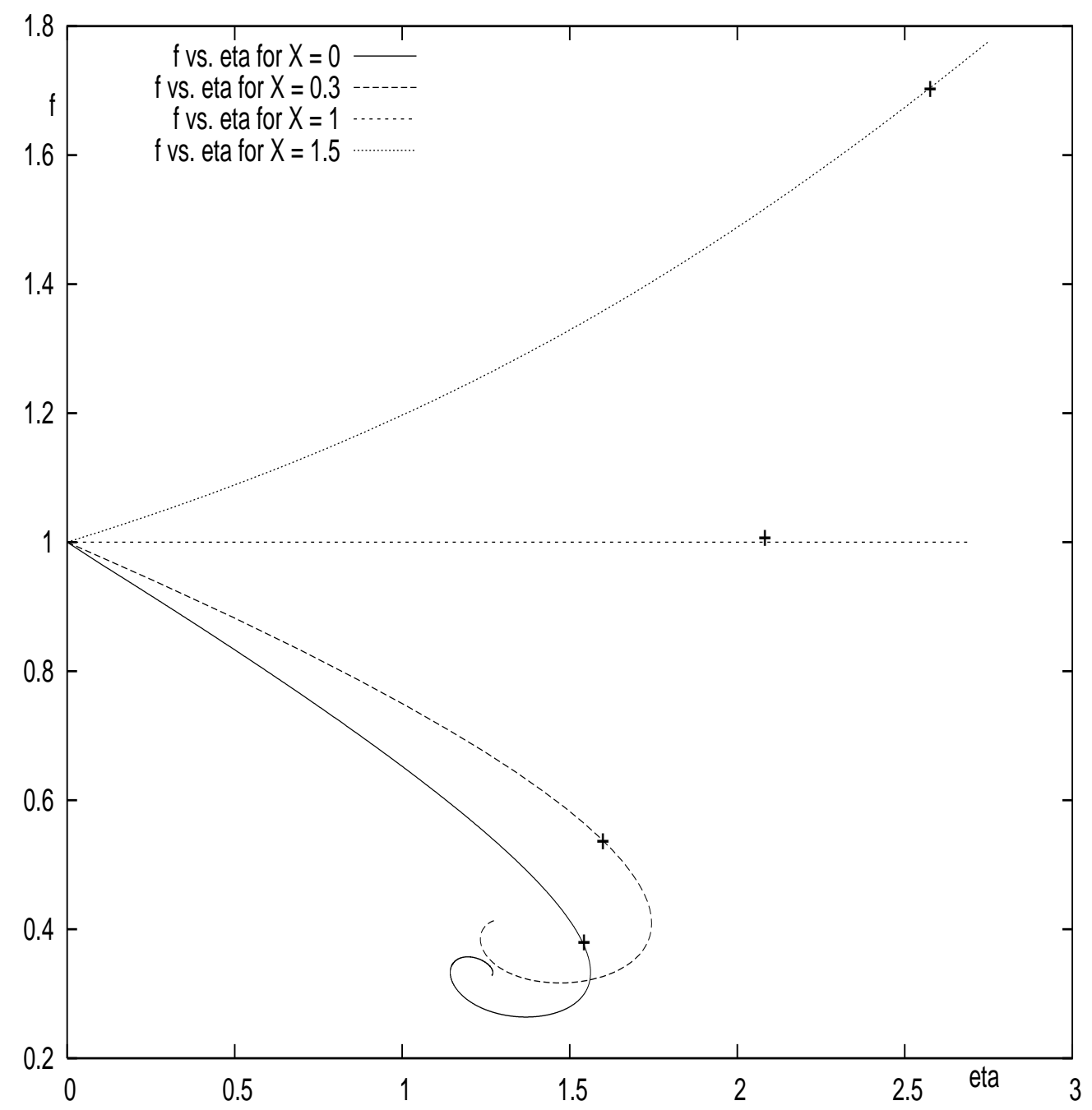

FIG. 1: The density at the boundary $f(X, \eta)$ versus $\eta$ for $X=0,0.3,1,1.5$ by the mean field approach. The instability points $\eta_{o}(X)$ where the thermal compressibility diverges are pinpointed on the plot by a + . They are $\eta_{o}(X=0)=1.510 \ldots$, $\eta_{o}(X=0.3)=1.63 \ldots, \eta_{o}(X=1)=2.04 \ldots$ and $\eta_{o}(X=1.5)=2.55 \ldots$. The gas is stable from $\eta=0$ till $\eta=\eta_{o}(X)$ in the canonical ensemble.

\section{The isothermal sphere with $\Lambda \neq 0$}

For spherically symmetric configurations the mean field equation (2.8) becomes an ordinary non-linear differential equation.

$$
\frac{\mathrm{d}^{2} \Phi}{\mathrm{d} R^{2}}+\frac{2}{R} \frac{\mathrm{d} \Phi}{\mathrm{d} R}+4 \pi\left(\eta e^{\Phi(R)}-\xi\right)=0
$$

The various thermodynamic quantities are expressed in terms of their solutions. We work in a unit sphere, therefore the radial variable runs in the interval $0 \leq R \leq R_{\max }, R_{\max } \equiv\left(\frac{3}{4 \pi}\right)^{\frac{1}{3}}$. The density of particles $\rho(R)$ has to be normalized according to eq.(2.9). Integrating eq.(2.10) from $R=0$ to $R=R_{\max }$ yields,

$$
\eta-\xi=-R_{\max }^{2} \Phi^{\prime}\left(R_{\max }\right)
$$

Setting,

$$
\Phi(R)=u(x)+\ln \frac{\xi^{R}}{\eta^{R}} \quad, \quad x=\sqrt{3 \xi^{R}} \frac{R}{R_{\max }},
$$


$\xi^{R}=\frac{\xi}{R_{\max }}$ and $\eta^{R}=\frac{\eta}{R_{\max }}$, the saddle-point equation (2.10) simplifies as,

$$
\frac{\mathrm{d}^{2} u}{\mathrm{~d} x^{2}}+\frac{2}{x} \frac{\mathrm{d} u}{\mathrm{~d} x}+e^{u(x)}-1=0
$$

In order to have a regular solution at origin we have to impose

$$
u^{\prime}(0)=0 \text {. }
$$

We find from eqs. 2.11)- 2.12) for fixed values of $\eta$ and $\xi$,

$$
u^{\prime}\left(\sqrt{3 \xi^{R}}\right)=-\frac{\eta^{R}-\xi^{R}}{\sqrt{3 \xi^{R}}}
$$

Eqs.(2.14) and (2.15) provide the boundary conditions for the nonlinear ordinary differential equation (2.13). In particular, they impose the dependence of $u_{0} \equiv u(0)$ on $\eta^{R}$ and $\xi^{R}$.

We find from eq.(2.13) for small $x$ the expansion of $u(x)$ in powers of $x$ with the result,

$$
u(x)=u_{0}+\left(1-e^{u_{0}}\right) \frac{x^{2}}{6}+e^{u_{0}}\left(e^{u_{0}}-1\right) \frac{x^{4}}{120}+\mathcal{O}\left(x^{6}\right) .
$$

where we imposed eq.(2.14). The value of $u_{0}$ follows by imposing eq. (2.15).

Using eqs.(2.6), (2.7) and (2.12) we can express the density of particles in terms of the solution of eq.(2.13)

$$
\rho(R)=X \exp \left[u\left(\sqrt{3 X \eta^{R}} \frac{R}{R_{\max }}\right)\right] .
$$

We choose to express the physical quantities in terms of $\eta$ [eq.(2.5)] and the ratio $X$ of dark energy to self-gravity [eq.(2.6)]. The local pressure obeys the ideal gas equation of state in local form $P(R)=\frac{N T}{V} \rho(R)$. Using eqs.(2.17) the pressure at the boundary is given by

$$
P\left(R_{\max }\right)=\frac{N T}{V} X \exp \left[u\left(\sqrt{3 X \eta^{R}}\right)\right] \equiv \frac{N T}{V} f(X, \eta) .
$$

The contrast $C(X, \eta)$ between the pressure at the center and at the boundary can be written as,

$$
C(X, \eta) \equiv \frac{P(0)}{P\left(R_{\max }\right)}=e^{u_{0}-u\left(\sqrt{3 X \eta^{R}}\right)}
$$

We plot in fig. 1 the density at the boundary versus $\eta$ for different values of $X$.

\section{STABILITY OF THE SELF-GRAVITATING GAS}

\section{A. Stability for $\Lambda=0$.}

The self-gravitating system can be in one of two phases: gaseous or highly condensed. Mean field theory describes the gas phase and gives exactly the physical quantities in the thermodynamic limit. The equilibrium density $\rho(\vec{x})$ solution of the mean field equation (2.8) minimizes the Helmholtz free energy in the canonical ensemble. This density is the most probable distribution which becomes absolutely certain in the infinite $N$ limit. All thermodynamics quantities follows from this density $\rho(\vec{x})$. This continuous density $\rho(\vec{x})$ solution of the mean field equation (2.8) fails to describe the condensed phase.

The condensed phase have been found by Monte-Carlo simulations for $\Lambda=0$ [2]. In the microcanonical ensemble the center collapses in a very dense core surrounded by a halo of particles. In the canonical ensemble all the particles collapse in a very dense body [2].

The stability of the gaseous phase in the mean field approach can be analyzed looking for the extrema of the dimensionless parameter 10$]$

$$
\zeta=\frac{G^{3} m^{6} N^{2} P}{T^{4}}
$$




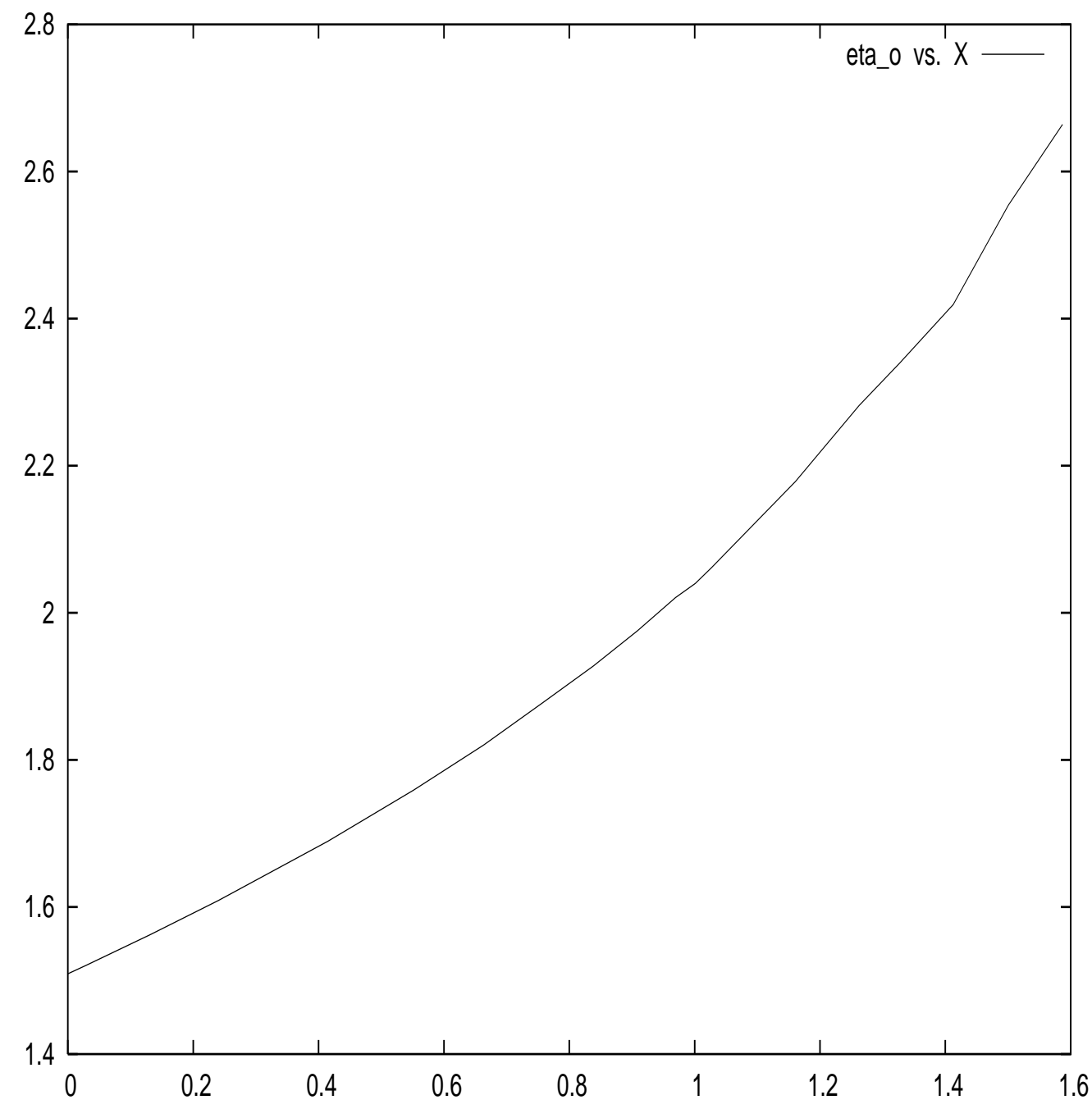

FIG. 2: The value of the parameter $\eta$ at the Jeans instability: $\eta=\eta_{o}(X)$ versus $X$.

when the temperature $T$, the pressure $P$ and the number of particles $N$ are kept fixed in the canonical ensemble. The gas phase becomes instable (for $\Lambda=0$ ) when $\zeta$ is maximal [10].

Using eqs. 2.5), (2.18) and (3.1) we find that

$$
\zeta=\eta^{3} f_{0}(\eta)
$$

where $f_{0}(\eta) \equiv f(X=0, \eta)$ is the external density in absence of dark energy.

A better physical insight is obtained by looking to the the isothermal compressibility

$$
K_{T}=-\frac{1}{V}\left(\frac{\partial V}{\partial P}\right)_{T} .
$$

Using eqs. (2.5) and (2.18) we find that the dimensionless isothermal compressibility $\kappa_{T} \equiv \frac{N T}{V} K_{T}$ takes the form

$$
\kappa_{T}=\frac{1}{f_{0}(\eta)+\frac{\eta}{3} f_{0}^{\prime}(\eta)} .
$$

We see from eqs.(3.2) and (3.4) that

$$
\kappa_{T}=\frac{3 \eta^{2}}{\frac{d \zeta}{d \eta}}
$$


Hence, $\kappa_{T}$ diverges at the extrema of $\zeta$.

The isothermal compressibility $\kappa_{T}$ is positive from $\eta=0$ till $\eta=\eta_{o}=1.510 \ldots$. At this point $\kappa_{T}$ as well as the specific heat at constant pressure diverge and change their signs [2, 7]. Moreover, at this point the speed of sound at the center of the sphere becomes imaginary $[\underline{3}$. Therefore, small density fluctuations will grow exponentially in time instead of exhibiting oscillatory propagation. Such a behaviour leads to the Jeans instability and collapse 14]. Monte-Carlo simulations confirmed the presence of this instability at $\eta=\eta_{o}=1.510 \ldots$ in the canonical ensemble for $\Lambda=0$ [2].

\section{B. Jeans instability for $\Lambda \neq 0$}

We now compute the dimensionless isothermal compressibility for the self-gravitating gas with $\Lambda \neq 0$. Introducing the dimensionless parameter

$$
\alpha=\frac{2 G^{3} m^{5} N^{2} \Lambda}{T^{3}}=\eta^{3} X
$$

the dimensionless isothermal compressibility $\kappa_{T}$ takes the form:

$$
\kappa_{T}=\frac{1}{f(\eta, \alpha)+\frac{\eta}{3}\left(\frac{\partial f}{\partial \eta}\right)(\eta, \alpha)} .
$$

The Jeans instability happens when the isothermal compressibility diverges. This happens for $\eta=\eta_{o}(X)$ verifying:

$$
f\left(\eta_{o}, \alpha\right)+\frac{\eta_{o}}{3}\left(\frac{\partial f}{\partial \eta}\right)\left(\eta_{o}, \alpha\right)=0
$$

Thus, for a fixed $\alpha, \eta_{o}$ is the value of $\eta$ which maximizes the quantity

$$
\zeta \equiv \eta^{3} f(\eta, \alpha)=\frac{G^{3} m^{6} N^{2} P}{T^{4}} .
$$

As for the self-gravitating gas with $\Lambda=0, \zeta$ is maximal when the specific heat at constant pressure diverges and changes its sign. We plot $\eta_{o}$ versus $X$ in fig. 2 using eq. (3.5). Monte Carlo simulations confirm that the onset of the Jeans collapse happens at $\eta=\eta_{o}$.

We can write $\eta[$ eq.(2.5)] as

$$
\eta=\frac{G m M}{T L}
$$

where $M$ is the total mass of the gas. Therefore, the collapse condition $\eta \geq \eta_{o}(X)$ can be written as

$$
M \geq M_{0} \eta_{o}(X) \quad \text { where } \quad M_{0} \equiv \frac{L T}{G m} .
$$

The gas collapses when its mass takes a larger value than the critical one $M_{J}(X) \equiv M_{0} \eta_{o}(X)$. We can consider $M_{J}(X)$ as the generalization of the Jeans mass in the presence of the cosmological constant. Notice that the presence of $\Lambda$ increases the Jeans mass with respect the $\Lambda=0$ case [see fig. 2 .

\section{PHYSICAL PICTURE}

\section{A. Behaviour of the gas phase with $\mathrm{X}$}

The effects of self-gravitation and dark energy go in opposite directions. Self-gravitating forces are attractive, while dark energy produces repulsion. If the ratio of dark energy and self-gravity is $X<1$ [defined by eq.(2.6)], self-gravitation dominates over dark energy. If $X=1$ the effect of self-gravitation is exactly compensated by the dark energy. If $X>1$ dark energy dominates over self-gravitation. We illustrate these three cases plotting $f(X, \eta)$ versus $\eta$ for $X=0.3,1,1.5$ in fig. [1] 


\section{- first case: $X<1$ : self-gravity dominates}

We illustrate this case choosing $X=0.3$. We see in fig. 团 that $f(X, \eta)$ versus $\eta$ exhibits a form analogous to $f(X=0, \eta)$ versus $\eta$ in absence of dark energy. $f(X, \eta)$ has two Riemann sheets as a function of $\eta$. In the first sheet $f(X, \eta)$ monotonically decreases with $\eta$ for fixed $X<1$.

- second case: $X=1$ : exact compensation

The effect of self-gravitation is compensated by the effect of dark energy. We see in fig. 1 that $f(X=1, \eta)=1$. An exactly homogeneous sphere $\rho(R)=1$ is a solution (see below). In such special case the self-gravitating gas behaves as a perfect gas (with $P V=N T$ everywhere).

- third case: $X>1$ : dark energy dominates

We illustrate this case choosing $X=1.5$. We see in fig. 1 that $f(X=1.5, \eta)$ is an increasing function of $\eta$ for fixed $X>1$.

The Jeans instability happens in the three cases. The value of $\eta=\eta_{o}(X)$ where the gas collapses increases with $X$ (fig. 2). The gas collapses for $X>0$ at a higher density $\frac{N}{V^{\frac{1}{3}}}$ for a given temperature or for a lower temperature for a given density $\frac{N}{V^{\frac{1}{3}}}$ than for $X=0$. That is, the domain of stability of the gas increases for increasing $X$. The dark energy has an anti-gravity effect that disfavours the collapse pushing the particles toward the boundary of the sphere. Notice that the point of Jeans instability is in the first Riemann sheet of the $\eta$-plane for $0 \leq X<1$.

\section{B. The particle density $\rho(R)$}

We study the density $\rho(R)$ as a function of the radial coordinate $R$. The density $\rho(R)$ of the self-gravitating gas in absence of dark energy $(X=0)$ is always a decreasing function of $R$. This follows from the attractive character of gravitation (see for example ref. [2, 3$]$ ).

The $R$-dependence of the density $\rho(R)$ of a self-gravitating gas in the presence of dark energy is more involved because the dark energy opposes to the attraction by gravity. The functions $\rho(R)$ and $u(x)$ have the same qualitative dependence in $R$ since they are related by exponentiation [see eq. [2.17)].

The behaviour at the center of the sphere $(R=0)$ is governed by the sign of $u_{0}$ since $u^{\prime}(0)=0$ [eq. (2.14)] and we find from eq.(2.16) that $u^{\prime \prime}(0)=\frac{1}{3}\left(1-e^{u_{0}}\right)$. Hence,

$$
\operatorname{sign}\left[u^{\prime \prime}(0)\right]=-\operatorname{sign}\left[u_{0}\right] .
$$

Therefore, $\rho(R)$ decreases (increases) at $R=0$ if $u_{0}>0\left(u_{0}<0\right)$.

The behaviour at the boundary of the sphere $\left(R=R_{\max }\right)$ is governed by the sign of $\eta-\xi$ since according to eq.(2.15)

$$
\operatorname{sign}\left[u^{\prime}\left(R_{\max }\right)\right]=-\operatorname{sign}\left[\eta^{R}-\xi^{R}\right]=\operatorname{sign}(X-1) .
$$

Therefore, $\rho(R)$ decreases (increases) at $R_{\max }$ if $X<1(X>1)$.

The particle density exhibits for the stable solutions (namely, for $\kappa_{T}>0$ ) one of the following behaviours illustrated in fig. [3]

- decreasing: $X<1$ and $u_{0}>0$. The density $\rho(R)$ decreases from the center of the sphere till the boundary. We plot in fig. 3 the density $\rho(R)$ versus $R$ for $X=0.3$ and $\eta=1$. The self-gravitation dominates over dark energy.

- increasing: $X>1$ and $u_{0}<0$. The density $\rho(R)$ increases from the center of the sphere till the boundary. We plot in fig. [3 the density $\rho(R)$ versus $R$ for $X=1.5$ and $\eta=1$. The dark energy dominates over self-gravitation.

- homogeneous: $X=1$ and $u_{0}=0$. The density is homogeneous: $\rho(R)=1$. The effect of self-gravity of particles is exactly compensated by the effect of dark energy. We plot in fig. 3 the density $\rho(R)$ versus $R$ for $X=1$ and $\eta=1$.

Eq. (2.8) for $X=1$ is similar to the equation that describes in two space dimensions (multi)-vortices in the GinsburgLandau or Higgs model in the limit between superconductivity of type I and II [17]. However, for the vortex case one has $\xi=\eta<0$ since like charges repel each other in electrodynamics while masses attract each other in gravity. This opposite nature of the forces is responsible of the fact that eq.(2.8) for $X=1$ only has trivial stable solutions while a host of non-trivial solutions appear in the vortex case 17. 


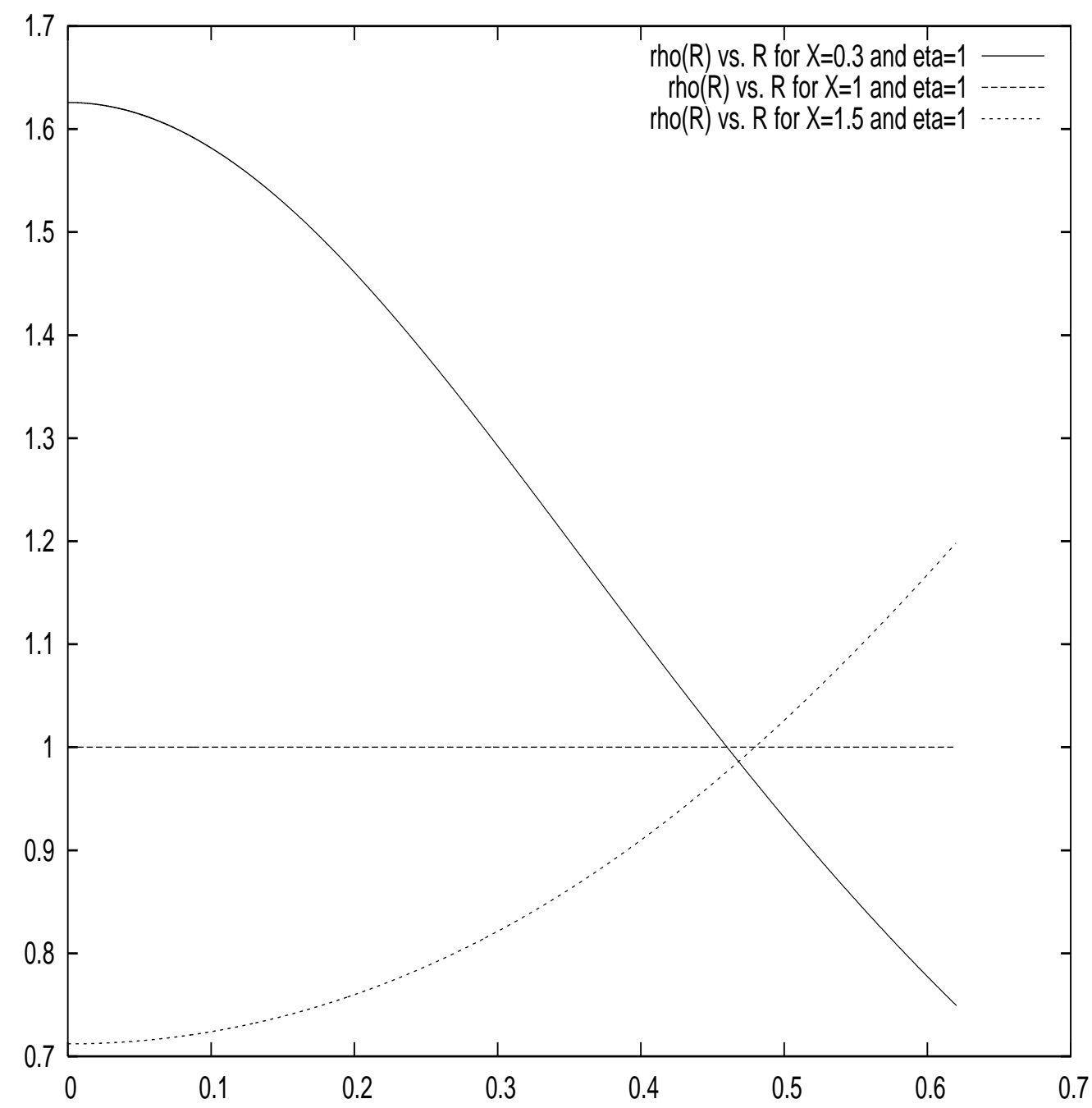

FIG. 3: The particle densities $\rho(R)$ vs. the radial coordinate $R$ for $X=0.3$ and $\eta=1$ (decreasing), $X=1$ and $\eta=1$ (homogeneous) and finally $X=1.5$ and $\eta=1$ (increasing) computed by the mean field approach

\section{MONTE CARLO CALCULATIONS}

\section{A. The Metropolis algorithm}

The standard Metropolis algorithm [16] was first applied to self-gravitating gas in absence of dark energy in ref. [2]. We apply this standard Metropolis to the self-gravitating gas in presence of dark energy. We perform it in a volume $V$ in the canonical ensemble at temperature $T$. We compute in this way the external pressure, the energy, the average density, the average particle distance and the average squared particle distance as function of $\eta$ for a given value of the ratio of dark energy and self-gravity $X \equiv \frac{\xi}{\eta}=\frac{2 \Lambda V}{m N}$.

We implement the Metropolis algorithm in the following way. We start from a random distribution of $N$ particles in the chosen volume. We update such configuration choosing a particle at random and changing at random its position. We then compare the energies of the former and the new configurations. We use the standard Metropolis test to choose between the new and the former configurations. The energy of the configurations are calculated performing the exact sums as in eq.(2.4). We use as statistical weight for the Metropolis algorithm in the canonical ensemble,

$$
e^{\eta u_{P}+\frac{2 \pi}{3} \xi u_{N}},
$$

[see eqs.(2.3) and (2.4)]. The number of particles go up to 2000 . 
We introduce a short distance cutoff in the Newtons' potential [see eq.(2.4)]

$$
u_{P} \equiv \frac{1}{N} \sum_{1 \leq i<j \leq N} \frac{1}{\left|\vec{r}_{i}-\vec{r}_{j}\right|_{A}},
$$

with

$$
\begin{aligned}
\left|\vec{r}_{i}-\vec{r}_{j}\right|_{A} & =\left|\vec{r}_{i}-\vec{r}_{j}\right| \quad, \quad \text { for } \quad\left|\vec{r}_{i}-\vec{r}_{j}\right|>A \\
& =A, \text { for } \quad\left|\vec{r}_{i}-\vec{r}_{j}\right|<A
\end{aligned}
$$

where $A \ll V^{\frac{1}{3}}$ is the short distance cut-off. The presence of the short distance cut-off prevents the collapse (here unphysical) of the self-gravitating gas (for more details see [2]).

\section{B. Influence of the geometry}

We apply the Metropolis algorithm to the self-gravitating gas in presence of dark energy in a sphere and in a cube. For $X=0$ (absence of dark energy) the Monte Carlo results in a sphere and in a cube with identical volume give practically the same results [2]. The geometry does not influence the physics of the system.

However, for $X>0$ (presence of dark energy) the Monte Carlo results in a sphere and in a cube turn to be different. This effect can be traced to the repulsive character of the dark energy that pushes the particles towards the boundary. As a result, the physical quantities becomes sensible to the geometry. We thus compared the mean field results with spherical symmetry from the previous sections and ref. [4] with Monte Carlo calculations performed on a sphere.

\section{Phase diagrams}

We recall that our self-gravitating system exhibits three different behaviours according the value of $X$, the relative rate of dark energy and self-gravity. In all three cases two different phases show up: for $\eta<\eta_{T}(X)$ we have a non perfect gas and for $\eta>\eta_{T}(X)$ it is a very condensed system with negative pressure. The transition between the two phases is very sharp.

- For $X<1$ the self-gravity dominates over dark energy. We plot the external density $f(X, \eta)=\frac{P V}{N T}$ versus $\eta$ for $X=0.3$ in fig. 4. In the gas phase $\frac{P V}{N T}$ monotonically decreases with $\eta$ from $\eta=0$ till the collapse point $\eta=\eta_{T}(X)$.

The average distance between particles $\left\langle\left|\vec{r}_{i}-\vec{r}_{j}\right|>\right.$ and the average squared distance between particles $\sqrt{<\left|\vec{r}_{i}-\vec{r}_{j}\right|^{2}>}$ monotonically decrease with $\eta$ (fig. 5). When the gas collapses at $\eta_{T},<\left|\vec{r}_{i}-\vec{r}_{j}\right|>$ and $\sqrt{<\left|\vec{r}_{i}-\vec{r}_{j}\right|^{2}>}$ exhibit a sharp drop.

- For $X=1$ the effect of dark energy exactly compensates the effect of self-gravity. We have $\frac{P V}{N T}=1$ in the gas phase. The system behaves like a perfect gas from $\eta=0$ till the collapse point $\eta=\eta_{T}(X)$.

- For $X>1$ the dark energy dominates over self-gravity. We plot in fig. [6 the external density $f(X, \eta)=\frac{P V}{N T}$ versus $\eta$ for $X=1.5$. In the gas phase $\frac{P V}{N T}$ monotonically increases with $\eta$ from $\eta=0$ till the collapse point $\eta=\eta_{T}(X)$.

We find that the Monte Carlo calculations in the sphere accurately reproduce the mean field results for $\frac{P V}{N T}$ in the gas phase with spherical symmetry. We then consider a cube and a sphere of identical volume. The Monte Carlo calculations turn to give different results in these two cases: a) The external pressure is larger in the cube than in the sphere. b) The gas phase is more stable in the cube. c) The collapse value $\eta_{T}(X)$ in the cube is larger than its value for the same $X$ in the sphere.

\section{The collapse point}

The phase transition happens in the Monte Carlo simulations at $\eta=\eta_{T}(X)$. It must be compared with the point of Jeans instability $\eta=\eta_{o}(X)$ according to mean field [see III.C] where the isothermal compressibility diverges and changes its sign. We see in that $\eta_{T}(X)$ is very close of $\eta_{o}(X)$. They are probably the same point. For $X=0.3$ 


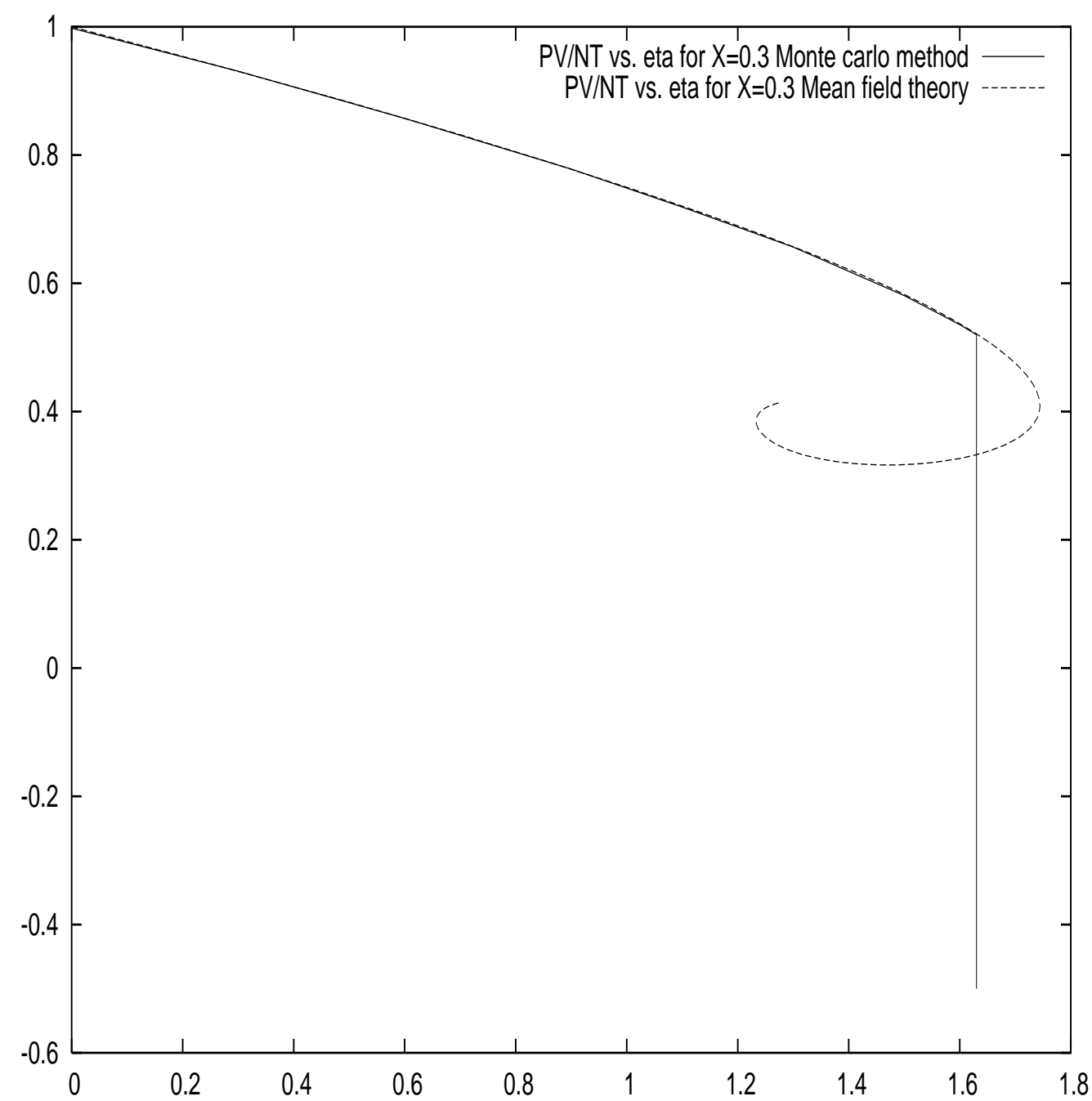

FIG. 4: The density at the boundary $f_{X}(\eta)=\frac{P V}{N T}$ versus $\eta$ for $X=0.3$ by Monte Carlo methods and mean field approach. The collapse value of the gas phase is $\eta_{T}(X=0.3)=1.63 \ldots$

we have $\eta_{o}(X)=1.63 \ldots$ and $\eta_{T}(X)=1.63 \ldots$ For $X=1.5$ we have $\eta_{o}(X)=2.55 \ldots$ and $\eta_{T}(X)=2.6 \ldots$ We conclude that the collapse of the system observed in the Monte Carlo simulations is due to the Jeans instability when the isothermal compressibility diverges and is well described by the mean field approach.

For $\eta \gtrsim \eta_{T}(X)$ we see that the gaseous phase is metastable. Before collapsing the system stay for a long Monte Carlo time in the metastable gaseous phase in the Monte Carlo computations. The Monte Carlo time for collapse increases with increasing value of $X$. This is due to the repulsive effect of dark energy. The gas phase is less unstable in the presence of dark energy.

\section{E. Average distribution of particles}

We illustrate the two behaviours of the self-gravitating gas in the presence of the cosmological constant plotting the average density as a function of two Cartesian coordinates, the third coordinate being fixed. For simplicity we depict the densities in the cube.

For $X>1$ we see that the density is larger on the boundary that at the center of the cube (fig. (7). The dark energy dominates over the self-gravity and pushes the particles toward the boundary of the cube.

For $X<1$ we see that the density is larger at the center than on the boundary of the cube (fig. 8). The self-gravity dominates over dark energy and attracts the particles to the center of the cube.

We illustrate now the two phases (gaseous and condensed) plotting the average particle distribution in a cube. Fig. 


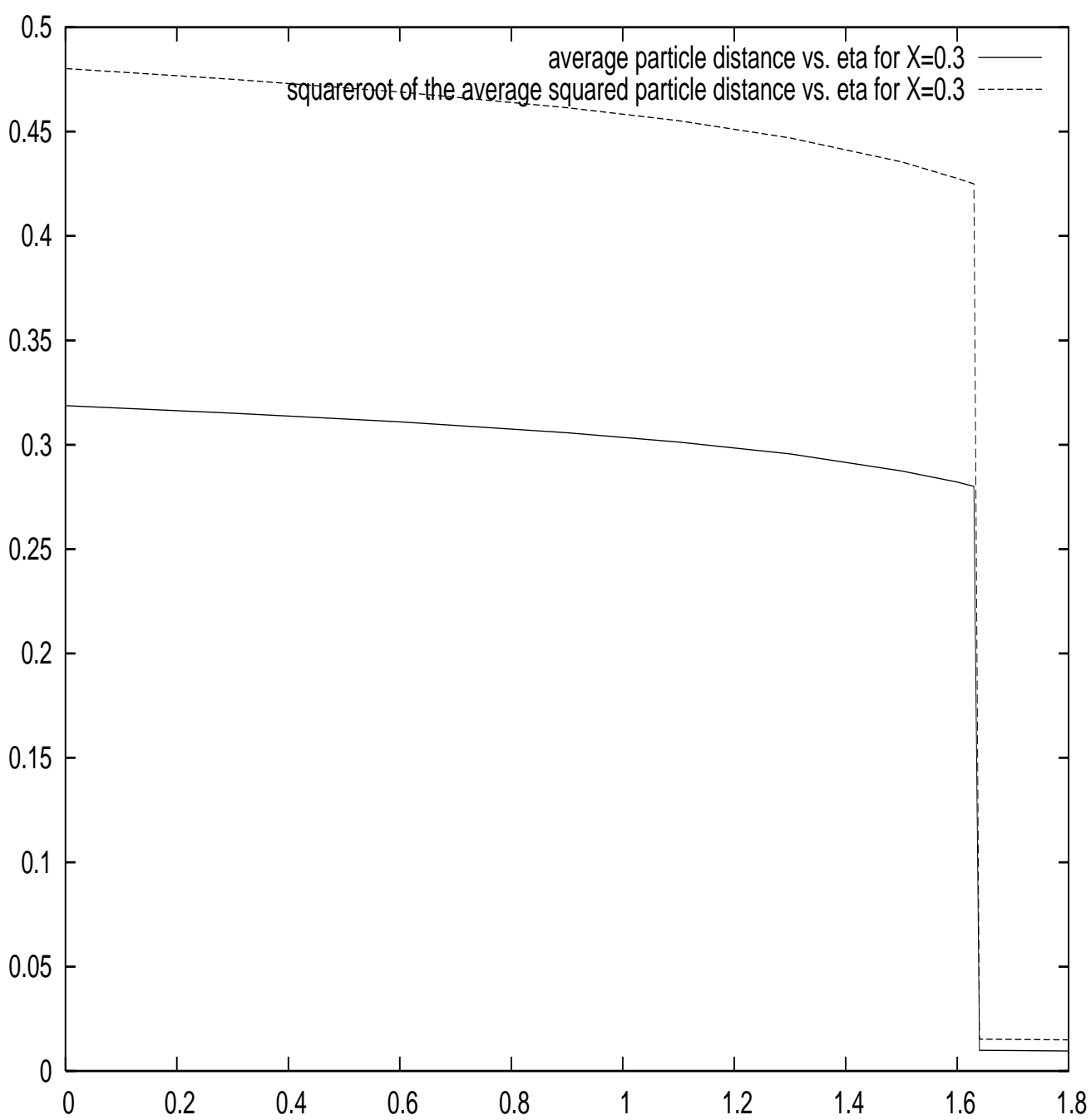

FIG. 5: The average particle distance and the average squared particle distance versus $\eta$ for $X=0.3$ by Monte Carlo methods. They exhibit a sharp decrease at the collapse value $\eta=\eta_{T}(X=0.3)=1.63 \ldots$.

9 and fig. 10] depict the average particle distribution from Monte Carlo calculations with 1000 particles for $X=0.3$ at both sides of the collapse point. Fig. 9 corresponds to the gaseous phase and fig. 10 to the collapsed phase.

We find that the Monte Carlo simulations (describing thermal equilibrium) are much more efficient than the $N$-body simulations integrating Newton's equations of motion. [Indeed, the integration of Newton's equations provides much more information than thermal equilibrium investigations]. Actually, a few hundreds of particles are enough to get quite accurate results in the Monte Carlo simulations (except near the collapse points). Moreover, the Monte Carlo results turns to be in excellent agreement with the mean field calculations up to corrections of the order $\frac{1}{N}$.

\section{F. The condensed phase}

In the condensed phase all the particles collapse in a very dense body (see fig. 10). The self-gravity contribution of the potential energy dominates overwhelmingly the dark energy contribution in the condensed phase. Using eqs. (2.2), (2.5) and (2.6) and the virial theorem [4] we obtain that the external pressure is expressed as

$$
f_{X}(\eta)=\frac{P V}{N T}=1-\frac{1}{3}\left\langle\frac{1}{\left|\vec{r}_{i}-\vec{r}_{j}\right|}\right\rangle \eta
$$




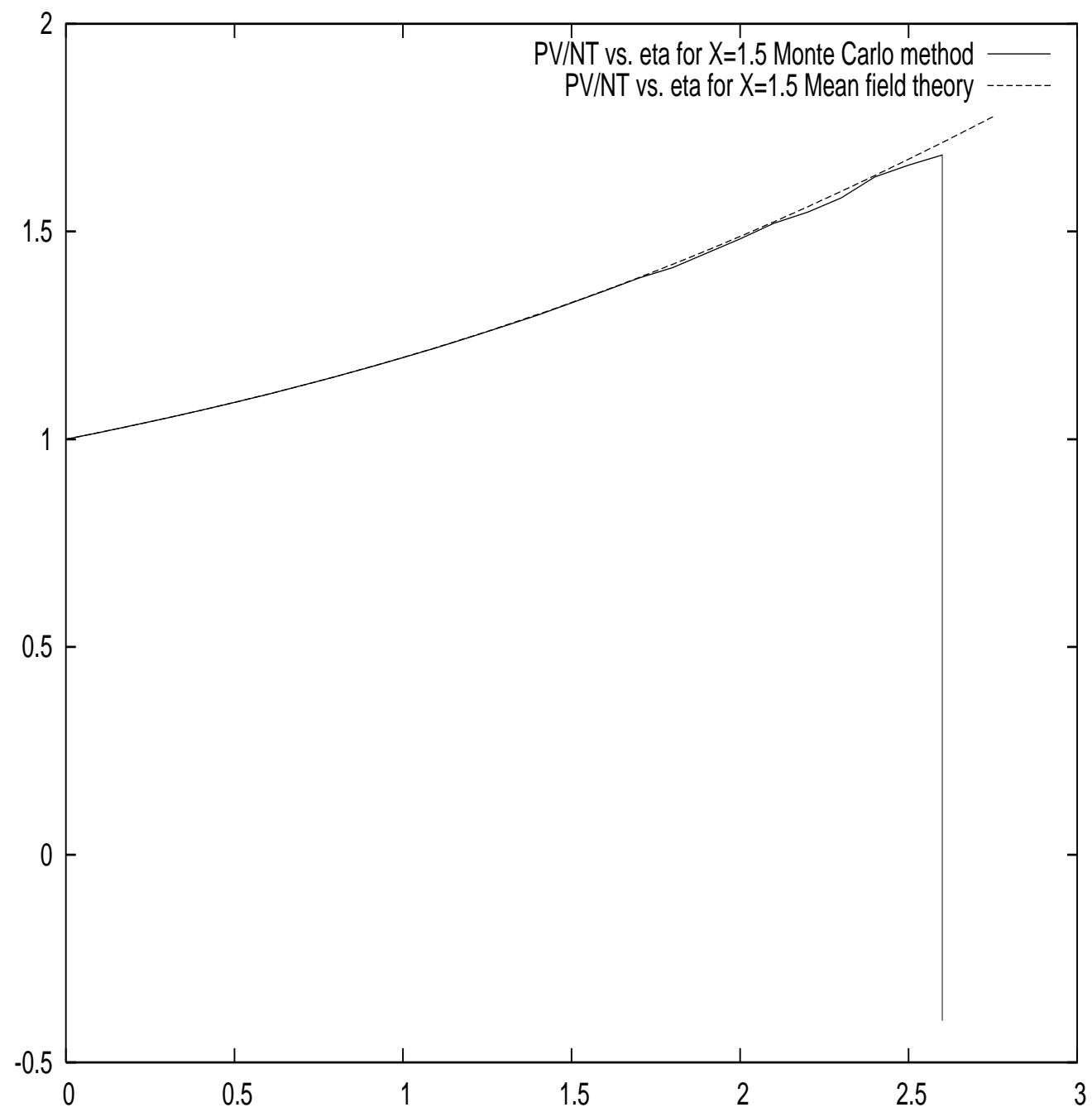

FIG. 6: The density at the boundary $f_{X}(\eta)=\frac{P V}{N T}$ versus $\eta$ for $X=1.5$ by Monte Carlo methods and mean field approach. The collapse value of the gas phase is $\eta_{T}(X=1.5)=2.6 \ldots$

The Monte Carlo results indicate that $\left\langle\frac{1}{\left|\vec{r}_{i}-\vec{r}_{j}\right|}\right\rangle \simeq 50$. Thus in this condensed phase the external pressure can be approximated by

$$
f_{X}(\eta)=\frac{P V}{N T}=1-K \eta
$$

where $K \simeq 16$.

Since $f(\eta)$ has a jump at the transition, the Gibbs free energy is discontinuous and we have a phase transition of the zeroth order as in the absence of cosmological constant [2].

\section{DISCUSSION AND CONCLUSIONS}

The behaviour of the self-gravitating gas is significantly influenced by the cosmological constant (or not) depending on the value of the ratio $X$ defined by eq.(2.6),

$$
X=\frac{2 \Lambda V}{m N}=2\left(\frac{\text { cosmological constant }}{\text { mass }}\right)_{V}=2 \frac{\Lambda}{\rho_{V}}
$$

where $\rho_{V} \equiv N m / V$ is the average mass density in the volume $V$ and where the subscript ()$_{V}$ indicates mass and cosmological constant inside the volume $V$. 
$X$ takes small values for astrophysical objects except for clusters of galaxies where Xgalaxyclusters $\sim 0.01$ and especially for the universe as a whole where Xuniverse $\sim 4$. The smallness of $X$ stems from the tiny value of the present cosmological constant $\Lambda=0.66310^{-29} \mathrm{~g} / \mathrm{cm}^{3}[18$. The particle mass density is much larger than $\Lambda$ in all situations except for the universe as a whole.

However, the non-relativistic and equilibrium treatment does not apply for the universe as a whole. Certainly, the galaxy distribution can be considered as a non-relativistic self-gravitating gas in the presence of the cosmological constant. But the gas of galaxies is certainly not yet at thermal equilibrium today. Perhaps, thermal equilibrium may be reached in some billions of years. Incidentally, at thermal equilibrium the particle density is regular at the center of the distribution in the whole range of $X$. Namely, there is no cusp at the center in a situation of thermal equilibrium.

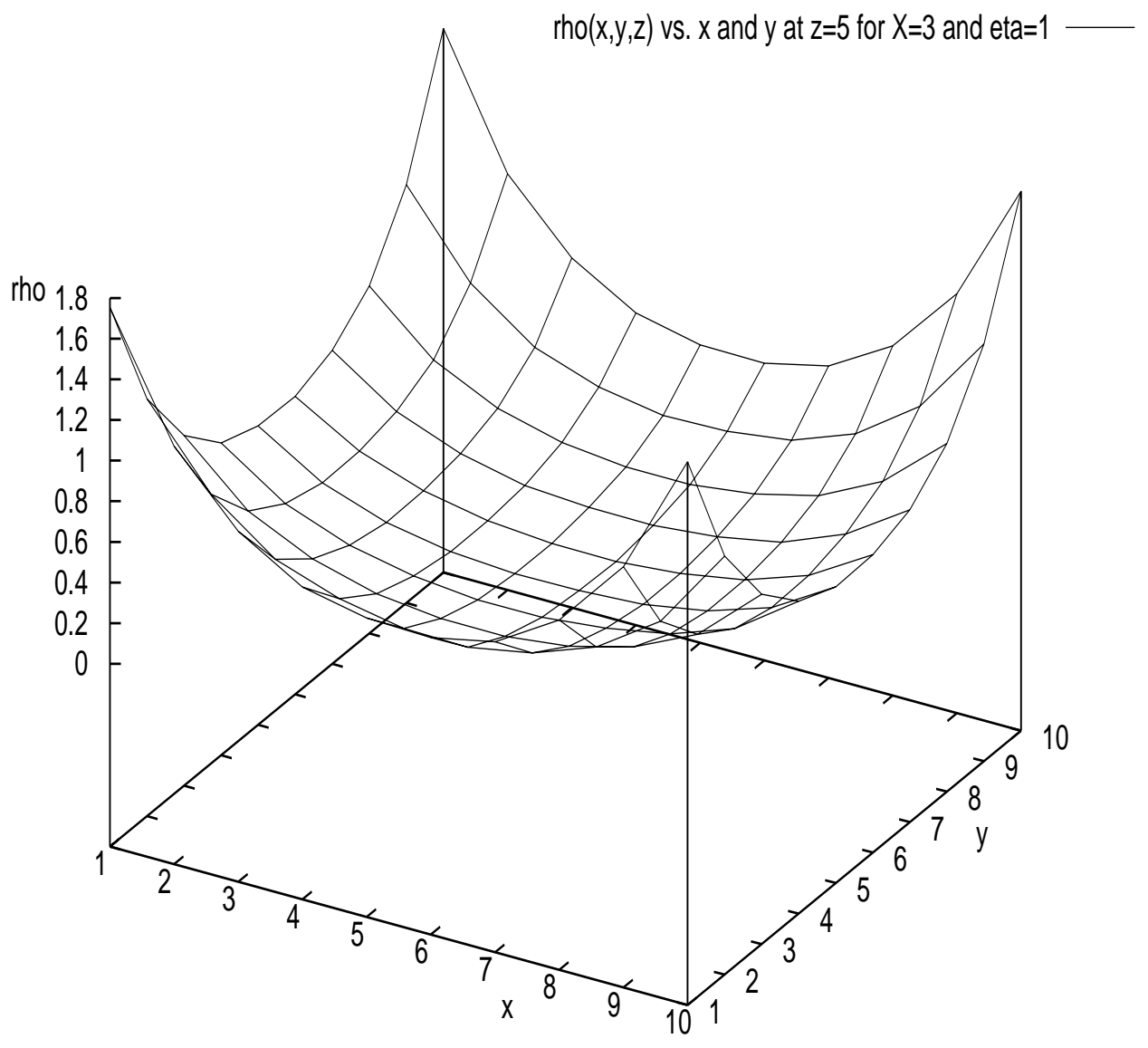

FIG. 7: The local density of the gas $\rho(x, y, z)$ versus the Cartesian coordinates $x$ and $y$ at $z=5$ in a cube of size 9 for $X=3$ and $\eta=1$ from Monte Carlo simulations. The density is larger on the boundary of the cube than at the center.

[1] P. J. E. Peebles, Principles of Physical Cosmology, Princeton (1993).

P. J. E. Peebles, B. Ratra, Revs. Mod. Phys. 75, 559 (2003).

[2] H. J. de Vega, N. Sánchez, Phys. Lett. B490, 180 (2000).

H. J. de Vega, N. Sánchez, Nucl. Phys. B 625, 409 (2002).

[3] H. J. de Vega, N. Sánchez, Nucl. Phys. B 625, 460 (2002).

[4] H. J. de Vega, J. A. Siebert, Nucl. Phys. B 707, 529 (2005). 


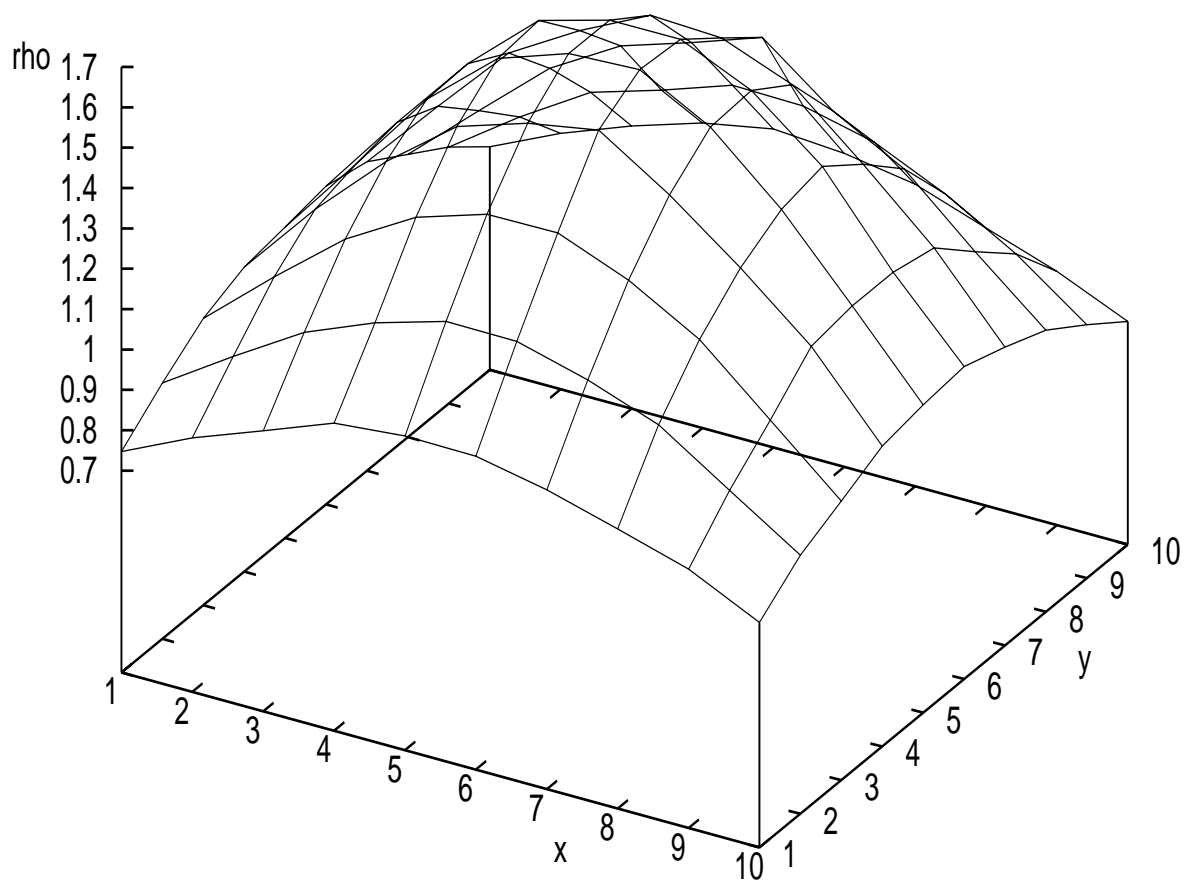

FIG. 8: The local density of the gas $\rho(x, y, z)$ versus the Cartesian coordinates $x$ and $y$ at $z=5$ in a cube of size 9 for $X=0.3$ and $\eta=1$ from Monte Carlo simulations. The density is larger at the center of the cube than on the boundary.

[5] R. Emden, Gaskugeln, Teubner, Leipzig und Berlin, 1907.

[6] S. Chandrasekhar, 'An introduction to the Study of Stellar Structure', Chicago Univ. Press, 1939.

[7] R. Ebert, Z. Astrophys. 37, 217 (1955).

[8] W.B.Bonnor, Mon. Not. R. astr. Soc. 116, 351 (1956).

[9] V. A. Antonov, Vest. Leningrad Univ. 7, 135 (1962).

[10] D. Lynden-Bell and R Wood, Mon. Not. R. astr. Soc. 138, 495 (1968).

[11] G. Horwitz and J. Katz, Ap. J. 211, 226 (1977) and 222, 941 (1978).

[12] T. Padmanabhan, Phys. Rep. 188, 285 (1990).

[13] W. C. Saslaw, 'Gravitational Physics of stellar and galactic systems', Cambridge Univ. Press, 1987.

[14] J. H. Jeans, Astronomy and cosmogony, Cambridge University Press (1919)

[15] G. Bertin, M. Trenti, ApJ, 584, 729, (2003).

[16] See, for example, K. Binder, D. W. Heerman, Monte-Carlo Simulations in Statisical Physics, Springer Series in Solid State, Vol.80, Berlin, 1988.

[17] H. J. de Vega and F. A. Schaposnik, Phys. Rev. D14, 1100 (1976). A. González Arroyo, A. Ramos, JHEP 0407, 008 (2004).

[18] S. Perlmutter et al., ApJ, 517, 565 (1999).

A. G. Riess et al., Astron. J, 1161009 (1998).

See for a review, B. P. Schmidt in Phase Transitions in the Early Universe: Theory and Observations, NATO ASI, Edited by H J de Vega, I M Khalatnikov and N. Sánchez, Series II, vol. 40, Kluwer, 2001. 


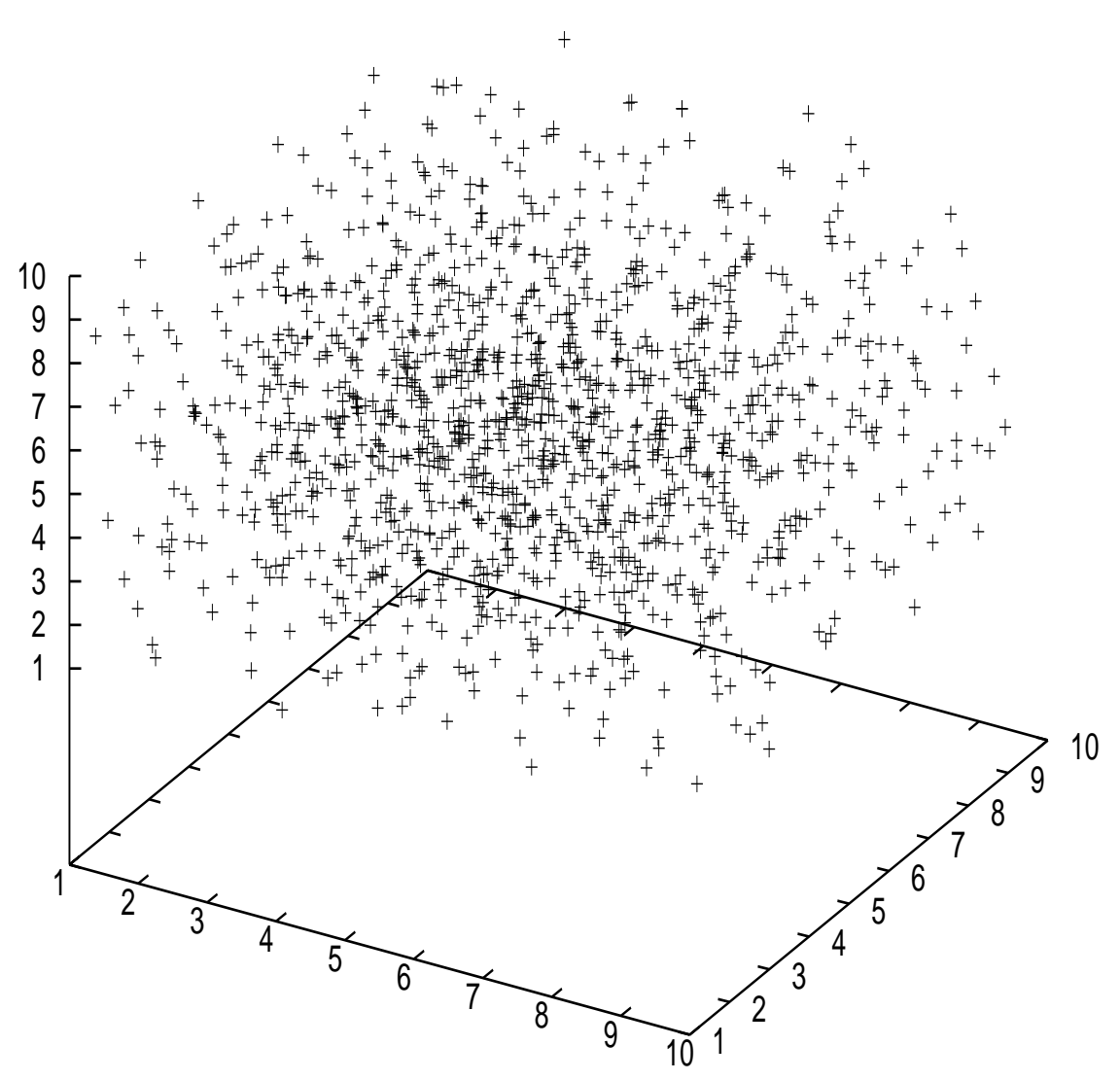

FIG. 9: Average particle distribution in the gaseous phase from Monte Carlo simulations in a cubic volume for $X=0.3, \eta=1.6$ and $N=2000$. A + denotes one particle. 


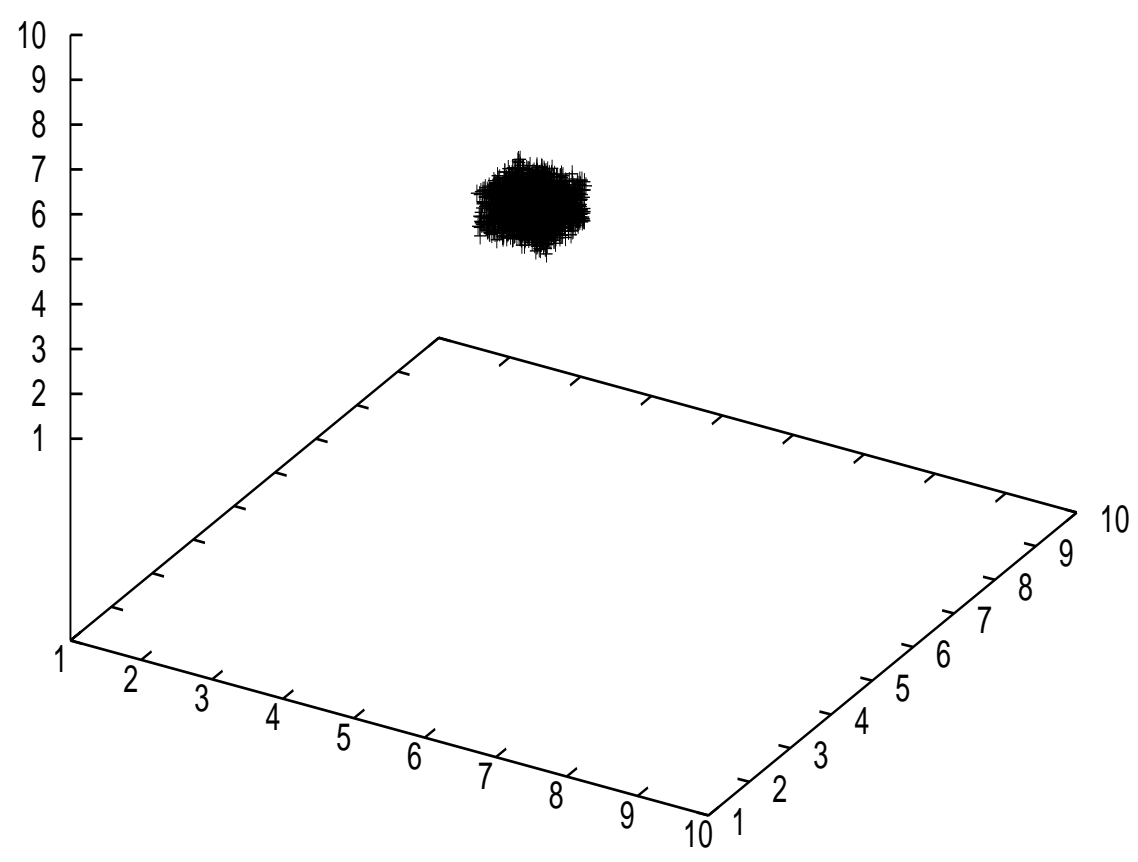

FIG. 10: Average particle distribution in the collapsed phase from Monte Carlo simulations in a cubic volume for $X=0.3$, $\eta=1.8$ and $N=2000$. A + denotes one particle. 